\title{
Boomerang Attack on Step-Reduced SHA-512
}

\author{
Hongbo $\mathrm{Yu}^{\star}$, Dongxia Bai \\ Department of Computer Science and Technology, \\ Tsinghua University, Beijing 100084, China \\ yuhongbo@mail.tsinghua.edu.cn \\ baidx10@mails.tsinghua.edu.cn
}

\begin{abstract}
SHA-2 (SHA-224, SHA-256, SHA-384 and SHA-512) is hash function family issued by the National Institute of Standards and Technology (NIST) in 2002 and is widely used all over the world. In this work, we analyze the security of SHA-512 with respect to boomerang attack. Boomerang distinguisher on SHA-512 compression function reduced to 48 steps is proposed, with a practical complexity of $2^{51}$. A practical example of the distinguisher for 48-step SHA-512 is also given. As far as we know, it is the best practical attack on step-reduced SHA-512.
\end{abstract}

Key words: SHA-512, hash functions, boomerang attack.

\section{Introduction}

Cryptographic hash functions play an important role in modern cryptology. In 2005, many notable hash functions, including MD5 and SHA-1, were broken by Wang et al. [32,33. Since these breakthrough results, many cryptographers have been convinced that these widely used hash functions can no longer be considered secure. Hash functions have been the target in lots of cryptanalytic attacks and cryptanalysis against hash functions has been improved significantly. People not only evaluate the three classical security requirements (preimage resistance, 2nd preimage resistance and collision resistance), but also consider all properties different from the expectation of a random oracle, such as (semi-) free-start collisions, near-collisions, boomerang distinguishers, etc. This is an important progress of the cryptanalysis for hash functions, since the security margin can be measured.

In recent years, the SHA-3 competition 23] organized by NIST has attracted more attention from the cryptographic community. However, as commonly used algorithms in many applications, SHA-2 still deserves

\footnotetext{
* Author is supported by 973 program (No. 2013CB834205), the National Natural Science Foundation of China (Nos. 61133013 and 61373142), the Tsinghua University Initiative Scientific Research Program (No. 20111080970).
} 
much detailed analysis to get a good view on its security. In this paper, we present boomerang attack on the reduced-step SHA-512.

Related Work. In the last few years, the security of SHA-256/512 against several attacks has been discussed in many papers. In [12] Isobe and Shibutani presented preimage attacks on SHA-256 and SHA-512 reduced to 24 steps. It was improved by Aoki et al. to 43-step SHA-256 and 46-step SHA-512 in [1]. Then Guo et al. gave advanced meet-in-the-middle preimage attacks on 42-step SHA-256/512 [10]. Later Khovratovich et al. applied biclique to preimages and extended attacks to 45 steps on SHA256 and 50 steps on SHA-512 [15]. Note that all these attacks only slightly faster than generic attack complexity $2^{256}$.

With respect to collision resistance, Mendel et al. presented the first collision attack on SHA-256 reduced to 18 steps in [21]. Then in [25] Nikolić and Biryukov improved the collision techniques and constructed a practical collision for 21 steps and a semi-free-start collision for 23 steps of SHA-256. This was later extended to 24 steps on SHA-256 and SHA512 by Sanadhya and Sarkar [26], and Indesteege et al. [11]. Then Mendel et al. improved the semi-free-start collisions on SHA-256 from 24 to 32 steps and gave a collision attack for 27 steps, which are all practical [19]. The best known collision attacks on SHA-256 so far are semi-free-start collisions for 38 and collisions for 31 out of 64 steps by Mendel et al. in [20. Recently, Eichlseder et al. presented semi-free-start collisions for SHA-512 on up to 38 steps in [8]. Compared with the preimage attacks, all these attacks have practical complexities.

At the rump session of Eurocrypt 2008, Yu and Wang presented nonrandomness of SHA-256 reduced to 39 steps [34, and gave a practical example of 33 steps. In [11, Indesteege et al. show nonrandom behavior of the SHA-256 compression function in the form of free-start near-collisions for up to 31 steps. In [17, Lamberger and Mendel gave a second-order differential collision on 46 steps of SHA-256 compression function. Later, Biryukov et al. extended the result in [17] by one round and presented a practical attack on 47 steps of SHA-256 in [7] in which the application of the attack strategy to SHA-512 was discussed, but no detailed differentials and example were given.

Our Contribution. In this work, the boomerang attack is used to show non-random properties for 48 (out of 80) steps of SHA-512 and an example of a confirming quartet is given. To the best of our knowledge, this is 
the best practical attack on reduced SHA-512. The summary of previous results and ours on SHA-512 are given in Table 1.

Table 1. Summary of the attacks on SHA-512

\begin{tabular}{c|c|c|c|c}
\hline attack type & target & steps & time & source \\
\hline \hline \multirow{4}{*}{ preimage attack } & $\mathrm{HF}$ & 24 & $2^{480}$ & {$[12]$} \\
\cline { 2 - 5 } & $\mathrm{HF}$ & 42 & $2^{501}$ & {$[1]$} \\
\cline { 2 - 5 } & $\mathrm{HF}$ & 46 & $2^{511.5}$ & {$[1]$} \\
\cline { 2 - 5 } & $\mathrm{HF}$ & 42 & $2^{494.6}$ & {$[10]$} \\
\cline { 2 - 5 } & $\mathrm{HF}$ & 50 & $2^{511.5}$ & {$[15]$} \\
\hline \multirow{3}{*}{ pseudo-preimage attack } & $\mathrm{HF}$ & 24 & $2^{480}$ & {$[12]$} \\
\cline { 2 - 5 } & $\mathrm{HF}$ & 46 & $2^{509}$ & {$[1]$} \\
\cline { 2 - 5 } & $\mathrm{HF}$ & 57 & $2^{511}$ & {$[15]$} \\
\hline \multirow{2}{*}{ collision } & $\mathrm{HF}$ & 24 & $2^{53}$ & {$[1]$} \\
\cline { 2 - 5 } & $\mathrm{HF}$ & 24 & $2^{22.5}$ & {$[26]$} \\
\hline semi-free-start collision & $\mathrm{HF}$ & 38 & $2^{40.5}$ & {$[8]$} \\
\hline boomerang attack & $\mathrm{CF}$ & 48 & $2^{51}$ & Sect. [4] \\
\hline
\end{tabular}

Outline. The structure of this paper is as follows. We give a short description of SHA-512 in Section 2. Section 3 summaries boomerang attack on hash functions. Then we present our boomerang attack on 48-step SHA-512 in Section 4. Finally, a conclusion of the paper is given in Section 5 .

\section{Description of SHA-2}

The SHA-2 (SHA-224, SHA-256, SHA-384 and SHA-512) hash function family is standardized by the National Institute of Standards and Technology (NIST), and adopts the Merkle-Damgård structure [22]. This section gives a short description of SHA-512. A complete specification can be found in [24].

SHA-512 is an iterated hash function that processes 1024-bit input message blocks and produces a 512-bit hash value. The compression function of SHA-512 consists of a message expansion function and a state update function.

The message expansion function splits the 1024-bit message block into 16 words $m_{i}, i=0, \ldots, 15$, and expands them into 8064 -bit message words $w_{i}$ as follows:

$$
w_{i}= \begin{cases}m_{i}, & 0 \leq i \leq 15 \\ \sigma_{1}\left(w_{i-2}\right)+w_{i-7}+\sigma_{0}\left(w_{i-15}\right)+w_{i-16}, & 16 \leq i \leq 79\end{cases}
$$


where the functions $\sigma_{0}(X)$ and $\sigma_{1}(X)$ are given by

$$
\begin{aligned}
& \sigma_{0}(X)=(X \ggg 1) \oplus(X \ggg 8) \oplus(X \gg 7), \\
& \sigma_{1}(X)=(X \ggg 19) \oplus(X \ggg 61) \oplus(X \gg 6) .
\end{aligned}
$$

The state update function updates 864 -bit chaining values $v_{i}=$ $\left(a_{i}, b_{i}, \ldots, h_{i}\right)$ in 80 steps using the 64 -bit word $w_{i}$ as follows:

$$
\begin{aligned}
& t_{1}=h_{i}+\Sigma_{1}\left(e_{i}\right)+F_{1}\left(e_{i}, f_{i}, g_{i}\right)+k_{i}+w_{i}, \\
& t_{2}=\Sigma_{0}\left(a_{i}\right)+F_{0}\left(a_{i}, b_{i}, c_{i}\right), \\
& a_{i+1}=t_{1}+t_{2}, b_{i+1}=a_{i}, c_{i+1}=b_{i}, d_{i+1}=c_{i}, \\
& e_{i+1}=d_{i}+t_{1}, f_{i+1}=e_{i}, g_{i+1}=f_{i}, h_{i+1}=g_{i},
\end{aligned}
$$

where $k_{i}$ is a step constant and the function $F_{0}, F_{1}, \Sigma_{0}, \Sigma_{1}$ are defined as follows:

$$
\begin{aligned}
& F_{0}(X, Y, Z)=(X \wedge Y) \oplus(Y \wedge Z) \oplus(X \wedge Z), \\
& F_{1}(X, Y, Z)=(X \wedge Y) \oplus(\neg X \wedge Z), \\
& \Sigma_{0}(X)=(X \ggg 28) \oplus(X \ggg 34) \oplus(X \ggg 39), \\
& \Sigma_{1}(X)=(X \ggg 14) \oplus(X \ggg 18) \oplus(X \ggg 41) .
\end{aligned}
$$

After 80 steps, the final hash value is computed by adding the output values to the initial state variables.

\section{Boomerang Distinguishers of Hash Functions}

The boomerang attack was introduced by Wagner in 1999 [31 against block ciphers. It treats the cipher as a cascade of two sub-ciphers, and uses short differentials in each sub-cipher. These differentials are combined in an adaptive chosen plaintext and ciphertext attack to exploit properties of the cipher that has high probability. Then Kelsey et al. [14] further developed it into a chosen plaintext attack called the amplified boomerang attack, and later it was developed by Biham et al. [4] into the rectangle attack. In [5], Biham et al. combined the boomerang (and the rectangle) attack with related-key differentials and proposed the related-key boomerang and rectangle attacks, which use the related-key differentials instead of the single-key differentials.

In recent years, the idea has been applied to hash functions as part of the new and useful hash function results. The first application presented in [13] used the idea of boomerang attack for the message modification technique in the collision attack for SHA-1. However, we note 
that this work does not build a boomerang property for a hash function to distinguish the hash functions from a random oracle, but only use the boomerang attack as a neutral bits tool for message modifications. The standard applications of boomerang attack to hash function were independently proposed by Biryukov et al. on BLAKE [6] and Lamberger and Mendel on the SHA-2 family [17]. In their works, the boomerang attacks are standalone distinguishers and work in the same way as for block ciphers - by producing the quartet of plaintexts and ciphertexts (input chaining values and output chaining values). Boomerang distinguishers have also been applied to SIMD-512 [18, HAVAL [27], RIPEMD [28], HAS-160 [29], Skein [935] and SM3 [16]3].

Let $H$ be the compression function of a hash function, $H_{0}$ and $H_{1}$ be two sub-ciphers: $H=H_{1} \circ H_{0}$. The boomerang attack for a compression function can be summarized as follows.

- Choose a random chaining value $v^{(1)}$ and a message $w^{(1)}$, compute $v^{(2)}=v^{(1)}+\beta, v^{(3)}=v^{(1)}+\gamma, v^{(4)}=v^{(3)}+\beta$ and $w^{(2)}=w^{(1)}+$ $\beta_{w}, w^{(3)}=w^{(1)}+\gamma_{w}, w^{(4)}=w^{(3)}+\beta_{w}$. We get a quartet $S=$ $\left\{\left(v^{(i)}, w^{(i)}\right) \mid i=1,2,3,4\right\}$.

- Compute backward from the quartet $S$ using $H_{0}^{-1}$ to obtain the initial values $I V_{1}, I V_{2}, I V_{3}$ and $I V_{4}$.

- Compute forward from the quartet $S$ using $H_{1}$ to obtain the output values $h_{1}, h_{2}, h_{3}$ and $h_{4}$.

- Check whether $I V_{2}-I V_{1}=I V_{4}-I V_{3}=\alpha$ and $h_{3}-h_{1}=h_{4}-h_{2}=\delta$ are fulfilled.

The complexity of the boomerang attack is summarized in [9135] as follows.

- Type I: A quartet satisfies $I V_{2}-I V_{1}=I V_{4}-I V_{3}=\alpha$ and $h_{3}-h_{1}=$ $h_{4}-h_{2}=\delta$ for fixed $\alpha$ and $\delta$. In this case, the generic complexity is $2^{n}$ where $n$ is the size of hash value.

- Type II: Only $h_{3}-h_{1}=h_{4}-h_{2}$ are required. This property is also called a second-order differential collision in [7]. In this case, the complexity for obtaining such a quartet is $2^{n / 3}$ using Wagner's generalized birthday attack [30].

- Type III: A quartet satisfies $I V_{2}-I V_{1}=I V_{4}-I V_{3}$ and $h_{3}-h_{1}=$ $h_{4}-h_{2}$. This property is also called a zero-sum distinguisher in [2]. In this case, the best known attack still takes time $2^{n / 2}$. 


\section{The Boomerang Attack on Reduced SHA-512}

In this section, we apply the boomerang attack to the SHA-512 compression function reduced to 48 steps. The basic idea of our attack is to connect two short differential paths in a quartet. The first step of our attack is to find two short differentials with high probabilities and the middle connection part in the middle does not contain any contradictions. Secondly, we derive the sufficient conditions for the messages and chaining variables for the steps in the middle. Thirdly, we satisfy the conditions in the middle steps by modifying the chaining variables and the message words. Finally, after the message modification, we search the right quartets that pass the verification of the distinguisher.

\subsection{Step-Reduced Differential Paths}

As shown in Table 2 and Table 3, we present the two differential paths used to construct the boomerang distinguisher on 48-step SHA-512, where the top differential path is from step 23 to 1 , and the bottom one is from step 24 to 48 . To describe the differential paths, we utilize the XOR difference $\Delta a=a \oplus a^{\prime}$, and $\Delta a: i(1 \leq i \leq 64)$ is used to denote that the $i$-th bit of $a$ is different from the $i$-th bit of $a^{\prime}$, and the rest of the bits of $a$ and $a^{\prime}$ are the same.

We start from the middle states of the distinguisher quartet $S$, and the differences of the message words $w_{i}$ and the chaining variables $v_{23}=$ $\left(a_{23}, b_{23}, \ldots, h_{23}\right)$ of the top differential path are selected as follows:

- $\Delta w_{7}: 64, \Delta w_{22}: 56,57,63, \Delta w_{i}=0(0 \leq i \leq 21, i \neq 7)$, if the top path has the differences in message words with this form, 18 steps (step 22 to 5) can be passed with probability 1 so that the path of this type has higher probability than any other ones not following this strategy.

- $\Delta a_{23}: 56,63, \Delta e_{23}: 56,63$, these differences are decided by the choice of differences of the message words above. In order to cancel the differences of message words, we derive the differences of all these chaining variables.

Now for the bottom differential path, we choose the differences as follows:

- $\Delta w_{23}: 41, \Delta w_{32}: 41, \Delta w_{47}: 33,40, \Delta w_{i}=0(24 \leq i \leq 46, i \neq 32)$, thus we can pass 14 steps (step 33 to 46) for free similarly.

- $\Delta b_{23}: 2,7,13,23,27,64, \Delta c_{23}: 41, \Delta e_{23}: 5,13, \Delta f_{23}: 2,7, \Delta g_{23}: 41$, $\Delta h_{23}=\Sigma_{1}\left(\Delta e_{23}\right)$, according to the differences of message words above and also considering the compatibility with the top differential path 
in the middle steps, the differences of chaining variables in the bottom path can be derived with some sufficient conditions given in part of Table 6 and Table 7 .

Table 2. The top differential path used for boomerang attack on SHA512.

\begin{tabular}{|c|c|c|c|c|c|c|c|c|c|}
\hline step & $\Delta w_{i}$ & $\Delta a$ & $\Delta b$ & $\Delta c$ & $\Delta d$ & $\Delta e$ & $\Delta f$ & $\Delta g$ & $\Delta h$ \\
\hline & & 64 & & & $23,25,30,36,46,50$ & 0 & 0 & 28,36 & 25,30 \\
\hline 1 & & & 64 & & & $23,46,50$ & & & 28,36 \\
\hline 2 & & & & 64 & & & $23,46,50$ & & \\
\hline 3 & & & & & 64 & & & $23,46,50$ & \\
\hline 4 & & & & & & 64 & & & $23,46,50$ \\
\hline 5 & & & & & & & 64 & & \\
\hline 6 & & & & & & & & 64 & \\
\hline 7 & & & & & & & & & 64 \\
\hline 8 & 64 & & & & & & & & \\
\hline $9-22$ & & & & & & & & & \\
\hline 23 & $56,57,63$ & 56,63 & & & & 56,63 & & & \\
\hline
\end{tabular}

Table 3. The bottom differential path used for boomerang attack on SHA-512.

\begin{tabular}{|c|c|c|c|c|c|c|c|c|c|}
\hline step & $\Delta w_{i}$ & $\Delta a$ & $\Delta b$ & $\Delta c$ & $\Delta d$ & $\Delta e$ & $\Delta f$ & $\Delta g$ & $\Delta h$ \\
\hline 23 & 33,40 & & $2,7,13,23,27,64$ & 41 & & 5,13 & 2,7 & 41 & $\Sigma_{1}\left(\Delta e_{23}\right)$ \\
\hline 24 & 41 & & & $2,7,13,23,27,64$ & 41 & & 5,13 & 2,7 & 41 \\
\hline 25 & & 41 & & & $2,7,13,23,27,64$ & & & 5,13 & 2,7 \\
\hline 26 & & & 41 & & & $23,27,64$ & & & 5,13 \\
\hline 27 & & & & 41 & & & $23,27,64$ & & \\
\hline 28 & & & & & 41 & & & $23,27,64$ & \\
\hline 29 & & & & & & 41 & & & $23,27,64$ \\
\hline 30 & & & & & & & 41 & & \\
\hline 31 & & & & & & & & 41 & \\
\hline 32 & & & & & & & & & 41 \\
\hline 33 & 41 & & & & & & & & \\
\hline \multicolumn{10}{|l|}{$34-47$} \\
\hline 48 & 33,40 & 33,40 & 0 & 0 & 0 & 33,40 & 0 & 0 & 0 \\
\hline
\end{tabular}

\subsection{Message Differences}

Let $w_{i}^{(1)}$ and $w_{i}^{(2)}(0 \leq i \leq 15)$ be two 1024-bit messages whose differences are shown in Table 2. In order to carry out the message modification in 
the middle steps (steps 23-32), we also need to determine the specific differences $w_{i}^{(1)} \oplus w_{i}^{(2)}(23 \leq i \leq 32)$.

For convenience, let $\Delta w_{i}^{(1,2)}$ denote the XOR difference of $w_{i}^{(1)}$ and $w_{i}^{(2)}$. According to the message expansion, we can compute the message differences $\Delta w_{i}^{(1,2)},(22 \leq i \leq 47)$ as follows.

$$
\begin{aligned}
\Delta w_{22}^{(1,2)} & =\left(\sigma_{1}\left(w_{20}^{(1)}\right)+w_{15}^{(1)}+\sigma_{0}\left(w_{7}^{(1)}\right)+w_{8}^{(1)}\right) \oplus\left(\sigma_{1}\left(w_{20}^{(2)}\right)+w_{15}^{(2)}+\sigma_{0}\left(w_{7}^{(2)}\right)+w_{8}^{(2)}\right) \\
\Delta w_{23}^{(1,2)} & =\left(\sigma_{1}\left(w_{21}^{(1)}\right)+w_{16}^{(1)}+\sigma_{0}\left(w_{8}^{(1)}\right)+w_{9}^{(1)}\right) \oplus\left(\sigma_{1}\left(w_{21}^{(2)}\right)+w_{16}^{(2)}+\sigma_{0}\left(w_{8}^{(2)}\right)+w_{9}^{(2)}\right) \\
\ldots & =\ldots \\
\Delta w_{47}^{(1,2)} & =\left(\sigma_{1}\left(w_{45}^{(1)}\right)+w_{40}^{(1)}+\sigma_{0}\left(w_{32}^{(1)}\right)+w_{31}^{(1)}\right) \oplus\left(\sigma_{1}\left(w_{45}^{(2)}\right)+w_{40}^{(2)}+\sigma_{0}\left(w_{32}^{(2)}\right)+w_{31}^{(2)}\right)
\end{aligned}
$$

Since $\sigma_{0}\left(w_{7}^{(1)}\right) \oplus \sigma_{0}\left(w_{7}^{(2)}\right)=\sigma_{0}\left(\Delta w_{7}^{(1,2)}\right)=0 x 4180000000000000$ and $\Delta w_{22}^{(1,2)}=0 x 4180000000000000$. The first equation holds if

$$
\begin{aligned}
& w_{22,56}^{(1)}=w_{7,57}^{(1)} \oplus w_{7,63}^{(1)} \oplus w_{7,64}^{(1)}, \\
& w_{22,57}^{(1)}=w_{7,58}^{(1)} \oplus w_{7,64}^{(1)} \oplus w_{7,1}^{(1)}, \\
& w_{22,63}^{(1)}=w_{7,64}^{(1)} \oplus w_{7,9}^{(1)} .
\end{aligned}
$$

In the same way, we set the differences $\Delta w_{i}^{(1,2)}(23 \leq i \leq 32)$ in the Table 4 and deduce the sufficient conditions on $w^{(1)}$ in Table 6 to meet the message expansion. Because we don't need to fulfill the message modifications in steps 34 to 48 , the message differences $\Delta w_{i}^{(1,2)}$ in these steps can keep free.

Table 4. Message differences in steps 23 to 33.

\begin{tabular}{|c|c|c|}
\hline$i$ & $\Delta w^{(1,2)}$ & $\Delta w^{(1,3)}$ \\
\hline 22 & 4180000000000000 & 0000008100000000 \\
\hline 23 & 8000000000000000 & 0000010000000000 \\
\hline 24 & 0502081000000002 & 0 \\
\hline 25 & 0200100000000004 & 0 \\
\hline 26 & 2804080001020010 & 0 \\
\hline 27 & 1008000002000020 & 0 \\
\hline 28 & $00825520891408 a 1$ & 0 \\
\hline 29 & $c 184220110080140$ & 0 \\
\hline 30 & 0504804080200408 & 0 \\
\hline 31 & 0001008000400800 & 0 \\
\hline 32 & $2 a b 100 a 291089050$ & 0000010000000000 \\
\hline
\end{tabular}


For the bottom path, the message differences $\Delta w_{i}^{(1,3)}, \Delta w_{i}^{(2,4)}(22 \leq$ $i \leq 47)$ are set in Table 3 . In order to get $w_{47}^{(3)}-w_{47}^{(1)}=w_{47}^{(4)}-w_{47}^{(2)}$, according to the message expansion

$$
w_{47}=w_{31}+\sigma_{0}\left(w_{32}\right)+w_{40}+\sigma_{1}\left(w_{45}\right),
$$

the following three equations must be satisfied.

$$
\begin{aligned}
& w_{32,41}^{(1)} \oplus w_{32,48}^{(1)} \oplus w_{32,47}^{(1)}=w_{32,41}^{(2)} \oplus w_{32,48}^{(2)} \oplus w_{32,47}^{(2)} \\
& w_{32,34}^{(1)} \oplus w_{32,41}^{(1)} \oplus w_{32,40}^{(1)}=w_{32,34}^{(2)} \oplus w_{32,41}^{(2)} \oplus w_{32,40}^{(2)} \\
& w_{32,35}^{(1)} \oplus w_{32,42}^{(1)} \oplus w_{32,41}^{(1)}=w_{32,35}^{(2)} \oplus w_{32,42}^{(2)} \oplus w_{32,41}^{(2)}
\end{aligned}
$$

The message difference $\Delta w_{32}^{(1,2)}$ we selected in Table 4 happens to meet the equations (4)-(6). Otherwise, we can adjust it.

Extend the messages $w_{i}^{(3)}$ and $w_{i}^{(4)}(22 \leq i \leq 47)$ in the backward direction. If we want to get $\Delta w_{i}^{(1,3)}=\Delta w_{i}^{(2,4)}(0 \leq i \leq 22)$, the following three equations must be satisfied.

$$
\begin{aligned}
& w_{22,56}^{(3)}=w_{7,57}^{(3)} \oplus w_{7,63}^{(3)} \oplus w_{7,64}^{(3)} \\
& w_{22,57}^{(3)}=w_{7,58}^{(3)} \oplus w_{7,64}^{(3)} \oplus w_{7,1}^{(3)} \\
& w_{22,63}^{(3)}=w_{7,64}^{(3)} \oplus w_{7,9}^{(3)}
\end{aligned}
$$

\subsection{Message Modification}

Here message modification technique [33] can be used to modify the message words and chaining variables to satisfy the conditions of the middle steps to significantly improve the complexity of our attack.

For the middle steps (23 to 33) of the boomerang distinguisher, by modifying some certain message words and chaining variables, we can fulfill all the conditions of one side and part of conditions of the other side of the bottom path. After the message modification, the conditions of step 23 in the top differential path can hold with probability 1, and the conditions of steps 24 to 33 in the bottom can hold with probability at least $2^{-40}$.

\subsection{Sketch of the Attack}

We divide our attack into two phases: the first phase is to find the right message words $w_{22}^{(1)}, \ldots, w_{32}^{(1)}$ and chaining variables $v_{23}^{(1)}$ so that the bottom 
paths of both sides in steps 23 to 33 hold; the second phase is to search $w_{17}^{(1)}, \ldots, w_{21}^{(1)}$ so that we can find a distinguisher quart. The sketch of attack is as follows.

1. Randomly select eleven 64-bit message words $w_{i}^{(1)}(22 \leq i \leq 32)$, and a 512-bit chaining variables $v_{23}^{(1)}=\left(a_{23}^{(1)}, b_{23}^{(1)}, \ldots, h_{23}^{(1)}\right)$. Modify the messages $w_{i}^{(1)}(22 \leq i \leq 32)$ to meet the conditions in Table 6. Compute $v_{i}^{(1)}(23 \leq i \leq 33)$. Modify $v_{23}^{(1)}$ and $w_{i}^{(1)}(22 \leq i \leq 32)$ so that $v_{i}^{(1)}$ $(24 \leq i \leq 33)$ satisfy all the conditions in Table 7 .

2. Let $w_{i}^{(2)}=w_{i}^{(1)} \oplus \Delta w_{i}^{(1,2)}, w_{i}^{(3)}=w_{i}^{(1)} \oplus \Delta w_{i}^{(1,3)}, w_{i}^{(4)}=w_{i}^{(2)} \oplus \Delta w_{i}^{(1,3)}$ $(22 \leq i \leq 32)$. The message differences $\Delta w_{i}^{(1,2)}$ and $\Delta w_{i}^{(1,3)}$ are defined in Table 4. Compute $v_{i}^{(j)}(j=2,3,4 ; 23 \leq i \leq 33)$. Check whether $v_{33}^{(1)} \oplus v_{33}^{(3)}=v_{33}^{(2)} \oplus v_{33}^{(4)}=0$. If yes, goto the next step. Otherwise, go back to step 1.

3. Select five 64-bit message words $w_{i}^{(1)}(17 \leq i \leq 21)$ randomly. Let $w_{i}^{(2)}=w_{i}^{(1)}(17 \leq i \leq 21)$. Compute $w_{i}^{(1)}$ and $w_{i}^{(2)}(33 \leq i \leq 47$, $0 \leq i \leq 16)$ in forward and backward directions separately. Let $w_{i}^{(3)}=$ $w_{i}^{(1)}$ and $w_{i}^{(4)}=w_{i}^{(2)}$ when $33 \leq i \leq 37$. Compute $w_{i}^{(3)}$ and $w_{i}^{(4)}$ when $38 \leq i \leq 47$ and $0 \leq i \leq 21$ by the message expansion.

4. Compute $v_{22}^{(j)}, v_{21}^{(j)}, \ldots, v_{0}^{(j)}(j=1,2,3,4)$ in backward direction and $v_{34}^{(j)}, v_{35}^{(j)}, \ldots, v_{48}^{(j)}(j=1,2,3,4)$ in forward direction. Check whether $v_{0}^{(2)}-v_{0}^{(1)}=v_{0}^{(4)}-v_{0}^{(3)}$ and $v_{48}^{(2)}-v_{48}^{(1)}=v_{48}^{(4)}-v_{48}^{(3)}$. If yes, output $w_{i}^{(j)}(j=1,2,3,4 ; 0 \leq i \leq 15)$ and $v_{1}^{(j)}(j=1,2,3,4)$. Otherwise, go to step 3 .

\subsection{Complexity of the Attack}

Based on the two differential paths and the message modification technique, we construct a 48-step boomerang distinguisher for SHA-512 compression function. The middle steps (23 to 33) of the boomerang distinguisher hold with probability $2^{-40}$. Besides, the probability of steps 22 to 1 of the top differential path is about $2^{-45}$ and for steps 34 to 48 of the bottom path is 1 . The probability of the message expansion is $2^{-6}$. Hence, the complexity of the 48 -step attack is $2^{40}+2^{45} \times 2^{6} \approx 2^{51}$ if we only get a zero-sum distinguisher, while the generic one is $2^{256}$.

The practical complexity of our attack leads to a practical boomerang distinguisher on up to 48-step compression function of SHA-512, and we 
are able to find its corresponding boomerang quartets. An example of 48step boomerang distinguisher for SHA-512 compression function is given in Table 5 .

Table 5. Example of a quart satisfying $H\left(I V^{(3)}, M^{(3)}\right)-H\left(I V^{(1)}, M^{(1)}\right)$ $-H\left(I V^{(4)}, M^{(4)}\right)+H\left(I V^{(2)}, M^{(2)}\right)=0$ for 48 steps of the SHA-512 compression function.

\begin{tabular}{|c|c|}
\hline (1) & d51d68d22cd614bb ad109f079123bc43 3e30194750de9356 b \\
\hline & $2788083 c 8 a f 206 a 4$ f53a6844e79ca3ff 83333924 f0fb45ee aeca4 \\
\hline & $9123 b c 43 b e$ \\
\hline . & a788083c8af206a4 753a4844e79ca3ff 0333591cf87b45ee 2ec84edfead0f3c1 \\
\hline$(3)$ & a7cc2ed702 d28a0787d13ece62 aaa0ccee3 \\
\hline 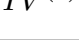 & e3ed3c3d8 9 \\
\hline (4) & bca41aa7cc2ed702 d28a0787d13ece62 2aa0c \\
\hline & $2 e 3 e d 3 c 3 d 81$ \\
\hline & 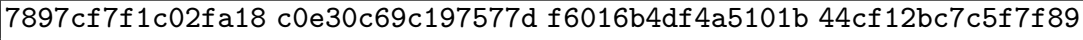 \\
\hline$M^{(1)}$ & $12 \mathrm{a} 41160 \mathrm{f}$ \\
\hline & 8bd715591505422b 82f9e09643a6f94e 8ae783224a988778 d858b794e8b95a4a \\
\hline & $211 \mathrm{f} 08 \mathrm{~b} 5 \mathrm{e} 33$ \\
\hline & 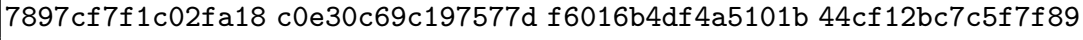 \\
\hline$M^{(2)}$ & d28a43112a41160f a481e26554edd575 8a \\
\hline & $5591505422 b 8$ \\
\hline & d98d2e211f08b5e3 3185a2321c2013d0 493b7695ecb8bc63 40dde2bb03f050f7 \\
\hline & b5e9b2bae2 da67703373f8f947 c \\
\hline & $361 d 1 a c c 84$ 49208682435343aa 8a4c7b5efe34b2e8 d6bcd7d0a70c5663 \\
\hline & 123cadba871 a134d5cebfae6e21 e32 \\
\hline & 9d4d5849a78a6aa9 4634d6dd6a193ca7 783f014e5106c88e bcd2f996a68b63f7 \\
\hline & 2ec928b5e9b2bae2 da67703373f8f947 c4c2b463d9c34453 a4d359b70a54809d \\
\hline$M T-1$ & acc84 49208682435343aa 8a4c7b5efe34b2e8 \\
\hline & $23 c a d b a 871$ a134d5cebfae6e21 e32 \\
\hline & 9d4d5849a78a6aa9 4634d6dd6a193ca7 783f014e51 \\
\hline
\end{tabular}

\section{Conclusion}

In this work, we propose two step-reduced differential paths with high probabilities of SHA-512 and build a boomerang distinguisher for the compression function of SHA-512 up to 48 steps out of 80 steps with practical complexity $2^{51}$, and the example of boomerang quartet is aslo presented. Our attack is the best practical result on SHA-512 to date. 


\section{References}

1. Aoki, K., Guo, J., Matusiewicz, K., Sasaki, Y., Wang, L.: Preimages for StepReduced SHA-2. In: Matsui, M. (ed.) ASIACRYPT 2009. LNCS, vol. 5912, pp. 578-597.

2. Aumasson, J.-P., Meier, W.: Zero-sum Distinguishers for Reduced Keccak$\mathrm{f}$ and for the Core Functions of Luffa and Hamsi, Available online, 2009. http://131002.net/data/papers/AM09.pdf.

3. Bai, D., Yu, H., Wang, G., Wang X.: Improved Boomerang Attacks on SM3. In: Boyd, C. and Simpson, L. (eds.) ACISP 2013. LNCS, vol. 7959, pp. 251-266.

4. Biham, E., Dunkelman, O., Keller, N.: The Rectangle Attack - Rectangling the Serpent. In: Pfitzmann, B. (ed.) EUROCRYPT 2001. LNCS, vol. 2045, pp. 340357.

5. Biham, E., Dunkelman, O., Keller, N.: Related-Key Boomerang and Rectangle Attacks. In: Cramer, R. (ed.) EUROCRYPT 2005. LNCS, vol. 3494, pp. 507-525.

6. Biryukov, A., Nikolić, I., Roy, A.: Boomerang Attacks on BLAKE-32. In: Joux, A. (ed.) FSE 2011. LNCS, vol. 6733, pp. 218-237.

7. Biryukov, A., Lamberger, M., Mendel, F., Nikolić, I.: Second-Order Differential Collisions for Reduced SHA-256. In: Lee, D.H., Wang, X. (ed.) ASIACRYPT 2011. LNCS, vol. 7073, pp. 270-287.

8. Eichlseder, A., Mendel F., Schläffer, M.: Branching Heuristics in Differential Collision Search with Applications to SHA-512. In: FSE 2014, accepted paper.

9. Gaëtan, L., Arnab R.: Boomerang Attacks on Hash Function Using Auxiliary Differentials. In: O. Dunkelman (ed.) CT-RSA 2012. LNCS, vol. 7178, pp. 215-230.

10. Guo, J., Ling, S., Rechberger, C., Wang, H.: Advanced Meet-in-the-Middle Preimage Attacks: First Results on Full Tiger, and Improved Results on MD4 and SHA-2. In: Abe, M. (ed.) ASIACRYPT 2010. LNCS, vol. 6477, pp. 56-75.

11. Indesteege, S., Mendel, F., Preneel, B., Rechberger, C.: Collisions and Other Nonrandom Properties for Step-Reduced SHA-256. In: Avanzi, R.M., Keliher, L., Sica, F. (eds.) SAC 2008. LNCS, vol. 5381, pp. 276-293.

12. Isobe, T., Shibutani, K.: Preimage Attacks on Reduced Tiger and SHA-2. In: Dunkelman, O. (ed.) FSE 2009. LNCS, vol. 5665, pp. 139-155.

13. Joux, A., Peyrin, T.: Hash Functions and the (Amplified) Boomerang Attack. In: Menezes, A. (ed.) CRYPTO 2007. LNCS, vol. 4622, pp. 244-263.

14. Kelsey, J., Kohno, T., Schneier, B.: Amplified Boomerang Attacks Against Reduced-Round MARS and Serpent. In: Schneier, B. (ed.) FSE 2000. LNCS, vol. 1978, pp. 75-93.

15. Khovratovich D., Rechberger C., Savelieva A.: Bicliques for Preimages: Attacks on Skein-512 and the SHA-2 Family. In: A. Canteaut (ed.) FSE 2012. LNCS, vol. 7549, pp. 244-263.

16. Kircanski, A., Shen, Y., Wang, G., Youssef, A.M.: 'Boomerang and SlideRotational Analysis of the SM3 Hash Function', In: Knudsen, L.R., Wu, H. (eds.) SAC 2012. LNCS, vol. 7707, pp. 304-320.

17. Lamberger, M., Mendel, F., Higher-Order Differential Attack on Reduced SHA256. Cryptology ePrint Archive: Report 2011/037. (2011).

18. Mendel, F., Nad, T.: 'Boomerang Distinguisher for the SIMD-512 Compression Function'. In: Bernstein, D.J., Chatterjee, S. (eds.) INDOCRYPT 2011. LNCS, vol. 7107, pp. 255-269.

19. Mendel, F., Nad, T., Schläffer, M.: Finding SHA-2 Characteristics: Searching Through a Minefield of Contradictions. In: D.H. Lee and X. Wang (eds.) ASIACRYPT 2011. LNCS, vol. 7073, pp. 288-307. 
20. Mendel, F., Nad, T., Schläffer, M.: Improving Local Collisions: New Attacks on Reduced SHA-256. In: T. Johansson and P. Nguyen (eds.) EUROCRYPT 2013. LNCS, vol. 7881, pp. 262-278.

21. Mendel, F., Pramstaller, N., Rechberger, C., Rijmen, V.: Analysis of Step-Reduced SHA-256. In: Robshaw, M.J.B. (ed.) FSE 2006. LNCS, vol. 4047, pp. 126-143.

22. Menezes, A.J., van Oorschot, P.C., Vanstone, S.A.: Handbook of Applied Cryptography. CRC Press, Boca Raton (1997).

23. National Institute of Standards and Technology: Announcing Request for Candidate Algorithm Nominations for a New Cryptographic Hash Algorithm (SHA-3) Family. Federal Register 27(212), 62212-62220 (November 2007).

24. National Institute of Standards and Technology: FIPS PUB 180-3: Secure Hash Standard. Federal Information Processing Standards Publication 180-3, U.S. Department of Commerce (October 2008).

25. Nikolić, I., Biryukov, A.: Collisions for Step-Reduced SHA-256. In: Nyberg, K. (ed.) FSE 2008. LNCS, vol. 5086, pp. 1-15.

26. Sanadhya, S.K., Sarkar, P.: New Collision Attacks Against up to 24-Step SHA-2. In: Chowdhury, D.R., Rijmen, V., Das, A. (eds.) INDOCRYPT 2008. LNCS, vol. 5365, pp. 91-103.

27. Sasaki, Y.: Boomerang Distinguishers on MD4-Based Hash Functions: First Practical Results on Full 5-Pass HAVAL. In: Miri, A., Vaudenay, S. (eds.) SAC 2011. LNCS, vol. 7118, pp. 1-18.

28. Sasaki, Y., Wang, L.: '2-Dimension Sums: Distinguishers Beyond Three Rounds of RIPEMD-128 and RIPEMD-160', http://eprint.iacr.org/2012/049.pdf, February 2012.

29. Sasaki, Y., Wang, L., Takasaki, Y., Sakiyama, K., Ohta, K.: 'Boomerang Distinguishers for Full HAS-160 Compression Function'. In: Hanaoka, G., Yamauchi, T. (eds.) IWSEC 2012. LNCS, vol. 7631, pp. 156-169.

30. Wagner, D.: A Generalized Birthday Problem. In: M. Yung (ed.) CRYPTO 2002. LNCS, vol. 2442, pp. 288-303.

31. Wagner, D.: The Boomerang Attack. In: Knudsen, L.R. (ed.) FSE 1999. LNCS, vol. 1636, pp. 156-170.

32. Wang, X., Yin, Y.L., Yu, H.: Finding Collisions in the Full SHA-1. In: Shoup, V. (ed.) CRYPTO 2005. LNCS, vol. 3621, pp. 17-36.

33. Wang, X., Yu, H.: How to Break MD5 and Other Hash Functions. In: Cramer, R. (ed.) EUROCRYPT 2005. LNCS, vol. 3494, pp. 19-35.

34. Wang, X., Yu, H.: Non-randomness of 39-step SHA-256. Presented at rump session of EUROCRYPT (2008).

35. Yu, H., Chen, J., Wang, X.: The Boomerang Attacks on the Round-Reduced Skein512. In: Kundsen, L.R., Wu, H. (eds.) SAC 2012. LNCS, vol. 7707, pp. 288-304.

\section{Appendix}


Table 6. The message conditions in $w_{22}^{(1)}-w_{32}^{(1)}$.

\begin{tabular}{|c|c|}
\hline & \\
\hline & $\begin{array}{l}w_{22,15}^{(1)}=w_{22,14}^{(1)}, w_{22,44}^{(1)}=w_{22,43}^{(1)} \oplus w_{22,35}^{(1)} \oplus w_{22,34}^{(1)} \oplus u \\
w_{22,14}^{(1)} \oplus w_{22,15}^{(1)} \oplus w_{22,6}^{(1)} \oplus w_{22,5}^{(1)}, w_{22,57}^{(1)}=w_{22,56}^{(1)} \oplus 1\end{array}$ \\
\hline$w_{23}$ & $\begin{array}{l}w_{23,41}^{(1)}=w_{22,33}^{(1)} \oplus w_{23,34}^{(1)} \oplus w_{23,40}^{(1)}, w_{23,42}^{(1)}=w_{23,40}^{(1)} \oplus w_{23,35}^{(1)} \oplus w_{23,34}^{(1)} \oplus 1, w_{23,48}^{(1)}=w_{23,40}^{(1)} \oplus \\
w_{23,41}^{(1)} \oplus w_{23,47}^{(1)} \oplus 1\end{array}$ \\
\hline$w_{24}^{(1)}$ & $\begin{array}{l}w_{24,2}^{(1)}=w_{22,21}^{(1)} \oplus w_{22,63}^{(1)} \oplus w_{22,8}^{(1)}, w_{24,37}^{(1)}=w_{22,57}^{(1)} \oplus w_{22,35}^{(1)} \oplus w_{22,44}^{(1)}, w_{24,37}^{(1)}=w_{22,57}^{(1)} \oplus \\
w_{22,35}^{(1)} \oplus w_{22,44}^{(1)}, w_{24,44}^{(1)}=w_{22,63}^{(1)} \oplus w_{22,41}^{(1)} \oplus w_{22,50}^{(1)}, w_{24,50}^{(1)}=w_{22,6}^{(1)} \oplus w_{22,48}^{(1)} \oplus w_{22,57}^{(1)} \\
w_{24,57}^{(1)}=w_{22,12}^{(1)} \oplus w_{22,54}^{(1)} \oplus w_{22,63}^{(1)}, w_{24,59}^{(1)}=w_{22,15}^{(1)} \oplus w_{22,57}^{(1)}\end{array}$ \\
\hline$w_{25}^{(1)}$ & $\begin{array}{l}w_{25,3}^{(1)}=w_{23,22}^{(1)} \oplus w_{23,64}^{(1)} \oplus w_{23,9}^{(1)}, w_{25,45}^{(1)}=w_{23,64}^{(1)} \oplus w_{23,42}^{(1)} \oplus w_{23,51}^{(1)}, w_{25,58}^{(1)}=w_{23,13}^{(1)} \oplus \\
w_{23,55}^{(1)} \oplus w_{23,64}^{(1)}\end{array}$ \\
\hline$w_{26}^{(1)}$ & $\begin{array}{l}w_{26,5}^{(1)}=w_{24,24}^{(1)} \oplus w_{24,2}^{(1)} \oplus w_{24,11}^{(1)}, w_{26,18}^{(1)}=w_{24,37}^{(1)} \oplus w_{24,15}^{(1)} \oplus w_{24,24}^{(1)}, w_{26,25}^{(1)}=w_{24,44}^{(1)} \oplus \\
w_{24,22}^{(1)} \oplus w_{24,31}^{(1)}, w_{26,44}^{(1)}=w_{24,63}^{(1)} \oplus w_{24,41}^{(1)} \oplus w_{24,50}^{(1)}, w_{26,51}^{(1)}=w_{24,6}^{(1)} \oplus w_{24,48}^{(1)} \oplus w_{24,57}^{(1)} \\
w_{26,60}^{(1)}=w_{24,15}^{(1)} \oplus w_{24,57}^{(1)}, w_{26,62}^{(1)}=w_{24,17}^{(1)} \oplus w_{24,59}^{(1)}\end{array}$ \\
\hline$w_{27}^{(1)}$ & $\begin{array}{l}w_{27,6}^{(1)}=w_{25,25}^{(1)} \oplus w_{25,3}^{(1)} \oplus w_{25,12}^{(1)}, w_{27,26}^{(1)}=w_{25,45}^{(1)} \oplus w_{25,23}^{(1)} \oplus w_{25,32}^{(1)}, w_{27,52}^{(1)}=w_{25,7}^{(1)} \oplus \\
w_{25,49}^{(1)} \oplus w_{25,58}^{(1)}, w_{27,57}^{(1)}=w_{27,19}^{(1)} \oplus w_{27,39}^{(1)} \oplus w_{27,6}^{(1)} \oplus w_{24,44}^{(1)} \oplus 1, w_{27,61}^{(1)}=w_{25,16}^{(1)} \oplus w_{25,58}^{(1)}\end{array}$ \\
\hline$w_{28}^{(1)}$ & $\begin{array}{l}w_{28,1}^{(1)}=w_{26,20}^{(1)} \oplus w_{26,62}^{(1)} \oplus w_{26,7}^{(1)}, w_{28,6}^{(1)}=w_{26,25}^{(1)} \oplus w_{26,3}^{(1)} \oplus w_{26,12}^{(1)}, w_{28,8}^{(1)}=w_{26,27}^{(1)} \oplus \\
w_{26,5}^{(1)} \oplus w_{26,14}^{(1)}, w_{28,12}^{(1)}=w_{26,31}^{(1)} \oplus w_{26,9}^{(1)} \oplus w_{26,18}^{(1)}, w_{28,19}^{(1)}=w_{26,38}^{(1)} \oplus w_{26,16}^{(1)} \oplus w_{26,25}^{(1)}, \\
\left.w_{28,21}^{(1)}=w_{26,40}^{(1)} \oplus w_{26,18}^{(1)} \oplus w_{26,27}^{(1)}, w_{28,25}^{(1)}=w_{26,44}^{(1)} \oplus w_{26,22}^{(1)} \oplus w_{26,31}^{(1)}, w_{28,28}^{(1)}=w_{26,47}^{(1)} \oplus\right) \\
w_{26,25}^{(1)} \oplus w_{26,34}^{(1)}, w_{28,32}^{(1)}=w_{26,51}^{(1)} \oplus w_{26,29}^{(1)} \oplus w_{26,38}^{(1)}, w_{28,38}^{(1)}=w_{26,57}^{(1)} \oplus w_{26,35}^{(1)} \oplus w_{26,44}^{(1)}, \\
\left.w_{28,41}^{(1)}=w_{26,60}^{(1)} \oplus w_{26,38}^{(1)} \oplus w_{26,47}^{(1)}, w_{28,43}^{(1)}=w_{26,62}^{(1)} \oplus w_{26,40}^{(1)} \oplus w_{26,49}^{(1)}, w_{28,45}^{(1)}=w_{26,64}^{(1)} \oplus\right) \\
w_{26,42}^{(1)} \oplus w_{26,51}^{(1)}, w_{28,47}^{(1)}=w_{26,2}^{(1)} \oplus w_{26,44}^{(1)} \oplus w_{26,53}^{(1)}, w_{28,50}^{(1)}=w_{26,5}^{(1)} \oplus w_{26,47}^{(1)} \oplus w_{26,56}^{(1)}, \\
w_{28,56}^{(1)}=w_{26,11}^{(1)} \oplus w_{26,53}^{(1)} \oplus w_{26,62}^{(1)}\end{array}$ \\
\hline$w_{29}^{(1)}$ & $\begin{array}{l}w_{29,7}^{(1)}=w_{27,26}^{(1)} \oplus w_{27,4}^{(1)} \oplus w_{27,13}^{(1)}, w_{29,9}^{(1)}=w_{27,28}^{(1)} \oplus w_{27,6}^{(1)} \oplus w_{27,15}^{(1)}, w_{29}^{(1)}=w_{22,56}^{(1)} \oplus w_{24,59}^{(1)}, \\
w_{29,15}^{(1)}=w_{22,57}^{(1)} \oplus w_{27,59}^{(1)} \oplus 1, w_{29,20}^{(1)}=w_{27}^{(1)} \oplus w_{27,39}^{(1)} \oplus w_{27}^{(1)}, w_{29,21}^{(1)}=w_{22,63}^{(1)} \oplus w_{24,2}^{(1)} \oplus \\
w_{29,8}^{(1)} \oplus 1, w_{29,29}^{(1)}=w_{27,48}^{(1)} \oplus w_{27,26}^{(1)} \oplus w_{27,35}^{(1)}, w_{29,33}^{(1)}=w_{27,52}^{(1)} \oplus w_{27,30}^{(1)} \oplus w_{27,39}^{(1)}, w_{29,42}^{(1)}= \\
w_{27,61}^{(1)} \oplus w_{27,39}^{(1)} \oplus w_{27,48}^{(1)}, w_{29,43}^{(1)}=w_{22,56}^{(1)} \oplus w_{24,37}^{(1)} \oplus w_{29,34}^{(1)}, w_{29,44}^{(1)}=w_{22,56}^{(1)} \oplus w_{29,34}^{(1)} \oplus w_{29,43}^{(1)} \oplus \\
w_{22,57}^{(1)} \oplus w_{29,35}^{(1)} \oplus 1, w_{29,46}^{(1)}=w_{27,1}^{(1)} \oplus w_{27,43}^{(1)} \oplus w_{27,52}^{(1)}, w_{29,47}^{(1)}=w_{22,56}^{(1)} \oplus w_{24,50}^{(1)} \oplus w_{29,5}^{(1)}, \\
w_{29,48}^{(1)}=w_{22,56}^{(1)} \oplus w_{29,6}^{(1)} \oplus w_{22,57}^{(1)} \oplus w_{29,5}^{(1)} \oplus w_{29,47}^{(1)}, w_{29,50}^{(1)}=w_{24,44}^{(1)} \oplus w_{29,41}^{(1)} \oplus w_{22,63}^{(1)}, \\
w_{29,51}^{(1)}=w_{27,6}^{(1)} \oplus w_{27,48}^{(1)} \oplus w_{27,57}^{(1)}, w_{29,54}^{(1)}=w_{24,57}^{(1)} \oplus w_{29,12}^{(1)} \oplus w_{22,63}^{(1)}, w_{29,55}^{(1)}=w_{24,57}^{(1)} \oplus \\
w_{29,13}^{(1)} \oplus w_{27,19}^{(1)} \oplus w_{27,61}^{(1)} \oplus 1, w_{29,56}^{(1)}=w_{22,56}^{(1)}, w_{29,57}^{(1)}=w_{22,57}^{(1)}, w_{29,58}^{(1)}=w_{29,6}^{(1)} \oplus w_{29,48}^{(1)} \oplus \\
w_{29,57}^{(1)} \oplus w_{29,7}^{(1)} \oplus w_{29,49}^{(1)} \oplus 1, w_{29,63}^{(1)}=w_{22,63}^{(1)}, w_{29,64}^{(1)}=w_{27,19}^{(1)} \oplus w_{27,61}^{(1)}\end{array}$ \\
\hline$w_{30}^{(1)}$ & $\begin{array}{l}w_{30,4}^{(1)}=w_{28,23}^{(1)} \oplus w_{28,1}^{(1)} \oplus w_{28,10}^{(1)}, w_{30,11}^{(1)}=w_{28,30}^{(1)} \oplus w_{28,8}^{(1)} \oplus w_{28,17}^{(1)}, w_{30,22}^{(1)}=w_{28,41}^{(1)} \oplus w_{28,19}^{(1)} \oplus \\
w_{28,28}^{(1)}, w_{30,32}^{(1)}=w_{28,51}^{(1)} \oplus w_{28,29}^{(1)} \oplus w_{28,38}^{(1)}, w_{30,33}^{(1)}=w_{30,11}^{(1)} \oplus w_{30,20}^{(1)} \oplus w_{30,32}^{(1)} \oplus w_{30,10}^{(1)} \oplus w_{30,19}^{(1)} \oplus \\
\left.1, w_{30,39}^{(1)}=w_{28,58}^{(1)} \oplus w_{28,36}^{(1)} \oplus w_{28,45}^{(1)}, w_{30,42}^{(1)}=w_{25,45}^{(1)} \oplus w_{25,3}^{(1)} \oplus w_{30,22}^{(1)} \oplus w_{30,9}^{(1)} \oplus w_{28,6}^{(1)} \oplus w_{28,48}^{(1)} \oplus\right) \\
w_{28,57}^{(1)}, w_{30,45}^{(1)}=w_{30,23}^{(1)} \oplus w_{30,32}^{(1)} \oplus w_{30,44}^{(1)} \oplus w_{30,22}^{(1)} \oplus w_{30,31}^{(1)} \oplus 1, w_{30,48}^{(1)}=w_{28,3}^{(1)} \oplus w_{28,45}^{(1)} \oplus \\
w_{28,54}^{(1)}, w_{30,51}^{(1)}=w_{28,6}^{(1)} \oplus w_{28,48}^{(1)} \oplus w_{28,57}^{(1)}, w_{30,52}^{(1)}=w_{30,30}^{(1)} \oplus w_{30,39}^{(1)} \oplus w_{30,51}^{(1)} \oplus w_{30,29}^{(1)} \oplus w_{30,38}^{(1)}, \\
w_{30,54}^{(1)}=w_{30,32}^{(1)} \oplus w_{30,41}^{(1)} \oplus w_{30,51}^{(1)} \oplus w_{30,29}^{(1)} \oplus w_{30,38}^{(1)} \oplus 1, w_{30,57}^{(1)}=w_{28,12}^{(1)} \oplus w_{28,54}^{(1)} \oplus w_{28,63}^{(1)}, \\
w_{30,59}^{(1)}=w_{28,14}^{(1)} \oplus w_{28,56}^{(1)}, w_{30,64}^{(1)}=w_{25,3}^{(1)} \oplus w_{30,22}^{(1)} \oplus w_{30,9}^{(1)} \oplus 1\end{array}$ \\
\hline$w_{31}^{(1)}$ & $\begin{array}{l}w_{31,12}^{(1)}=w_{29,31}^{(1)} \oplus w_{29,9}^{(1)} \oplus w_{29,18}^{(1)}, w_{31,23}^{(1)}=w_{29,42}^{(1)} \oplus w_{29,20}^{(1)} \oplus w_{29,29}^{(1)}, w_{31,40}^{(1)}=w_{29,59}^{(1)} \oplus \\
w_{29,37}^{(1)} \oplus w_{29,46}^{(1)}, w_{31,49}^{(1)}=w_{29,4}^{(1)} \oplus w_{29,46}^{(1)} \oplus w_{29,55}^{(1)}\end{array}$ \\
\hline $\begin{array}{l}(1) \\
32\end{array}$ & $\begin{array}{l}w_{32,5}^{(1)}=w_{30,24}^{(1)} \oplus w_{30,2}^{(1)} \oplus w_{30,11}^{(1)}, w_{32,7}^{(1)}=w_{30,26}^{(1)} \oplus w_{30,4}^{(1)} \oplus w_{30,13}^{(1)}, w_{32,13}^{(1)}=w_{30,33}^{(1)} \oplus \\
w_{30,11}^{(1)} \oplus w_{30,20}^{(1)}, w_{32,16}^{(1)}=w_{30,35}^{(1)} \oplus w_{30,13}^{(1)} \oplus w_{30,22}^{(1)}, w_{32,20}^{(1)}=w_{30,39}^{(1)} \oplus w_{30,17}^{(1)} \oplus w_{30,26}^{(1)}, \\
\left.w_{32,25}^{(1)}=w_{30,45}^{(1)} \oplus w_{30,23}^{(1)} \oplus w_{30,32}^{(1)}, w_{32,29}^{(1)}=w_{30,48}^{(1)} \oplus w_{30,26}^{(1)} \oplus w_{30,35}^{(1)}, w_{32,32}^{(1)}=w_{30,51}^{(1)} \oplus\right) \\
w_{30,29}^{(1)} \oplus w_{30,38}^{(1)} \oplus 1, w_{32,34}^{(1)}=w_{30,51}^{(1)} \oplus w_{30,29}^{(1)} \oplus w_{30,38}^{(1)} \oplus 1, w_{32,38}^{(1)}=w_{30,57}^{(1)} \oplus w_{30,35}^{(1)} \oplus w_{30,44}^{(1)}, \\
w_{32,40}^{(1)}=w_{30,59}^{(1)} \oplus w_{30,37}^{(1)} \oplus w_{30,46}^{(1)}, w_{32,47}^{(1)}=w_{23,47}^{(1)} \oplus 1, w_{32,49}^{(1)}=w_{30,4}^{(1)} \oplus w_{30,46}^{(1)} \oplus w_{30,55}^{(1)}, \\
\left.w_{32,53}^{(1)}=w_{30,8}^{(1)} \oplus w_{30,50}^{(1)} \oplus w_{30,59}^{(1)}, w_{32,54}^{(1)}=w_{30,9}^{(1)} \oplus w_{30,51}^{(1)} \oplus w_{30,60}^{(1)}, w_{32,56}^{(1)}=w_{30,11}^{(1)} \oplus\right) \\
w_{30,53}^{(1)} \oplus w_{30,62}^{(1)}, w_{32,58}^{(1)}=w_{27,58}^{(1)}, w_{32,60}^{(1)}=w_{30,15}^{(1)} \oplus w_{30,57}^{(1)}, w_{32,62}^{(1)}=w_{30,17}^{(1)} \oplus w_{30,59}^{(1)}\end{array}$ \\
\hline
\end{tabular}


Table 7. The conditions of chaining variables in the middle steps.

\begin{tabular}{|c|c|}
\hline steps & conditions \\
\hline & $a_{23,56}^{(1)}=w_{22,56}^{(1)} \oplus 1, a_{23,62}^{(1)}=w_{22,63}^{(1)} \oplus a_{23,3}^{(1)} \oplus w_{22,56}^{(1)} \oplus a_{23,4}^{(1)} \oplus a_{23,57}^{(1)}, a_{23,63}^{(1)}=w_{22,63}^{(1)}$ \\
\hline & $b_{23,41}^{(1)}=a_{23,41}^{(1)}$ \\
\hline 23 & $\begin{array}{l}c_{23,2}^{(1)}=a_{23,2}^{(1)}, c_{23,7}^{(1)}=a_{23,7}^{(1)}, c_{23,13}^{(1)}=a_{23,13}^{(1)}, c_{23,23}^{(1)}=a_{23,23}^{(1)}, c_{23,27}^{(1)}=a_{23,27}^{(1)}, c_{23,63}^{(1)}= \\
b_{23,63}^{(1)} \oplus 1, c_{23,64}^{(1)}=a_{23,64}^{(1)}\end{array}$ \\
\hline & $e_{23,13}^{(1)}=b_{23,13}^{(1)} \oplus 1, e_{23,56}^{(1)}=w_{22,56}^{(1)} \oplus 1, e_{23,63}^{(1)}=w_{22,63}^{(1)}$ \\
\hline & $f_{23,2}^{(1)}=b_{23,2}^{(1)} \oplus 1, f_{23,7}^{(1)}=b_{23,7}^{(1)} \oplus 1$ \\
\hline & $g_{23,41}^{(1)}=c_{23,41}^{(1)}, g_{23,63}^{(1)}=f_{23,63}^{(1)}$ \\
\hline 24 & $\begin{array}{l}a_{24,2}^{(1)}=b_{24,2}^{(1)}, a_{24,7}^{(1)}=b_{24,7}^{(1)}, a_{24,13}^{(1)}=b_{24,13}^{(1)}, a_{24,23}^{(1)}=b_{24,23}^{(1)}, a_{24,27}^{(1)}=b_{24,27}^{(1)}, a_{24,41}^{(1)}=b_{24,41}^{(1)}, \\
a_{24,63}^{(1)}=a_{23,63}^{(1)} \oplus 1, a_{24,64}^{(1)}=b_{24,64}^{(1)}\end{array}$ \\
\hline & $e_{24,2}^{(1)}=1, e_{24,5}^{(1)}=0, e_{24,7}^{(1)}=1, e_{24,13}^{(1)}=0$ \\
\hline 25 & $\begin{array}{l}a_{25,41}^{(1)}=h_{24,41}^{(1)}, a_{25,36}^{(1)}=a_{25,30}^{(1)} \oplus h_{24,41}^{(1)} \oplus h_{25,2}^{(1)} \oplus 1, a_{25,46}^{(1)}=a_{25,35}^{(1)} \oplus h_{24,41}^{(1)} \oplus h_{25,7}^{(1)} \oplus 1, \\
a_{25,52}^{(1)}=a_{25,47}^{(1)} \oplus h_{24,41}^{(1)} \oplus d_{25,13}^{(1)}\end{array}$ \\
\hline & $e_{25,5}^{(1)}=1, e_{25,13}^{(1)}=0, e_{25,23}^{(1)}=f_{25,23}^{(1)} \oplus 1, e_{25,27}^{(1)}=f_{25,27}^{(1)}, e_{25,64}^{(1)}=f_{25,64}^{(1)}$ \\
\hline 26 & $\begin{array}{l}e_{26,23}^{(1)}=d_{25,23}^{(1)}, e_{26,27}^{(1)}=d_{25,27}^{(1)}, e_{26,41}^{(1)}=c_{26,41}^{(1)}, e_{26,46}^{(1)}=e_{26,23}^{(1)} \oplus e_{26,19}^{(1)} \oplus e_{23,5}^{(1)} \oplus 1, \\
e_{26,54}^{(1)}=e_{26,31}^{(1)} \oplus e_{26,27}^{(1)} \oplus e_{23,13}^{(1)} \oplus 1, e_{26,64}^{(1)}=e_{26,37}^{(1)} \oplus e_{26,41}^{(1)} \oplus e_{26,23}^{(1)} \oplus g_{26,23}^{(1)} \oplus 1\end{array}$ \\
\hline 27 & $e_{27,23}^{(1)}=0, e_{27,27}^{(1)}=0, e_{27,64}^{(1)}=0$ \\
\hline & $a_{27,41}^{(1)}=b_{27,41}^{(1)}$ \\
\hline 28 & $e_{28,23}^{(1)}=1, e_{28,27}^{(1)}=1, e_{28,41}^{(1)}=f_{28,41}^{(1)}, e_{28,64}^{(1)}=1$ \\
\hline 29 & $\begin{array}{l}e_{29,41}^{(1)}=d_{28,41}^{(1)}, e_{29,45}^{(1)}=e_{29,41}^{(1)} \oplus e_{29,4}^{(1)} \oplus h_{29,27}^{(1)} \oplus 1, e_{29,18}^{(1)}=e_{29,14}^{(1)} \oplus d_{28,41}^{(1)} \oplus h_{29,64}^{(1)} \oplus 1, \\
e_{29,64}^{(1)}=e_{29,37}^{(1)} \oplus d_{28,41}^{(1)} \oplus h_{29,23}^{(1)} \oplus 1\end{array}$ \\
\hline 30 & $e_{30,41}^{(1)}=0$ \\
\hline 31 & $e_{31,41}^{(1)}=1$ \\
\hline
\end{tabular}

